\title{
Supply Chain Network Design for Critical Needs with Outsourcing
}

\author{
Anna Nagurney and Min Yu \\ Department of Finance and Operations Management \\ Isenberg School of Management \\ University of Massachusetts \\ Amherst, Massachusetts 01003 \\ Qiang Qiang \\ Management Division \\ Pennsylvania State University \\ Great Valley School of Graduate Professional Studies \\ Malvern, Pennsylvania 19355
}

August 2009; revised November 2009 and February 2010

Papers in Regional Science 90: (2011) pp 123-142.

\begin{abstract}
In this paper we consider the design of supply chain networks in the case of critical needs as may occur, for example, in disasters, emergencies, pending epidemics, and attacks affecting national security. By "critical needs" we mean products that are essential to the survival of the population, which can include, for example, vaccines, medicine, food, water, etc., depending upon the particular application. "Critical" implies that the demand for the product should be met as nearly as possible since otherwise there may be additional loss of life. The model that we develop captures a single organization, such as the government or a major health organization or corporation that seeks to "produce" the product at several possible manufacturing plants, have it stored, if need be, and distributed to the demand points. We assume that the organization is aware of the total costs associated with the various operational supply chain network activities, knows the existing capacities of the links, and is interested in identifying the additional capacity outlays, the production amounts, and shipment values so that the demand is satisfied with associated penalties if the demand is not met (as well as penalties with oversupply, which are expected to be lower). In addition, the organization has the option of outsourcing the production/storage/delivery of the critical product at a fixed/negotiated price and with the capacities of those entities being fixed and known. The solution of the model provides the optimal capacity enhancements and volumes
\end{abstract}


of product flows so as to minimize the total cost, which we assume to be a generalized cost, and can include time, subject to the demands being satisfied, as nearly as possible, under demand uncertainty.

Keywords: Supply chain design, networks, disasters, vaccine production, pandemics, emergency preparedness, humanitarian logistics, critical needs,demand uncertainty, healthcare, outsourcing 


\section{Introduction}

The number of disasters is increasing globally, as well as the people affected by disasters. At the same time, with the advent of increasing globalization, viruses are spreading more quickly and creating new challenges for medical and health professionals, researchers, and government officials. For example, between 2000 and 2004 the average annual number of disasters was 55\% higher than in the period 1994 through 1999, with $33 \%$ more humans affected in the former period than in the latter (cf. Balcik and Beamon (2008) and Nagurney and Qiang (2009)). The International Strategy for Disaster Reduction (2006) notes that approximately 150 million people required assistance, because of disasters, in 2005, with 157 million requiring assistance in 2006.

According to CNN.com (2009), as of July 31, 2009, the total number of deaths due to the H1N1 virus, also known as the swine flu, was 1,154, with an increase of 338 deaths since the World Health Organization's previous update on July 27, 2009, with 168 countries reporting cases. There has been progress on an H1N1 vaccine with the pharmaceutical giant GlaxoSmithKline signing contracts with nine governments to provide 96 million doses of a H1N1 vaccine come the Fall. Nevertheless, the pharmaceutical company is aware of possible capacity and production issues. Sanofi-Aventis (see Reuters.com (2009)), on the other hand, has begun large-scale production of an H1N1 vaccine at its plants in the US and in France. Clinical trials took place last summer. It will also be producing seasonal vaccines with its two Pennsylvania factories able to produce 150 million doses a year and its French plant 120 million doses of seasonal vaccine annually.

Vaccines are considered one of the most effective tools of public health policy, with current vaccination programs saving millions of lives a year and new vaccines enabling the protection of millions more, including those in some of the poorest countries in the world (see WHO (2009) and Mowery and Mitchell (1995)). Nevertheless, between 2000 and 2004 there were nationwide shortages in the US of six recommended childhood vaccines and the production of adult influenza vaccine was interrupted several times (Coleman et al. (2005)). Public health and medical professionals have long been cognizant of the vulnerability of vaccine production and delivery with the latter often relying on only one or two manufacturers for critical products (cf. Treanor (2004)). New illnesses, in turn, pose further stresses for vaccine 
(and medicine) development, production, and distribution.

Underlying the delivery of goods and services in times of crises, such as in the case of disasters, pandemics, and life-threatening major disruptions, are supply chains, without which essential products do not get delivered in a timely manner, with possible increased disease, injuries, and casualties. However, although the average number of disasters has been increasing annually over the past decade the average percentage of needs met by different sectors in the period 2000 through 2005 identifies significant shortfalls. According to Development Initiatives (2006), based on data in the Financial Tracking System of the Office for the Coordination of Humanitarian Affairs, from 2000-2005, the average needs met by different sectors in the case of disasters were: $79 \%$ by the food sector; $37 \%$ of the health needs; $35 \%$ of the water and sanitation needs; $28 \%$ of the shelter and non-food items, and $24 \%$ of the economic recovery and infrastructure needs. A case in point is the response to Hurricane Katrina, in which essential services failed completely, and which has been called an "American tragedy" (Guidotti (2006)). Additional challenges in providing essential goods and services in humanitarian logistics operations are faced by the developing world, whose infrastructure, even in the best of times, may be lacking (see Nagurney (2008)).

As a more recent example, on January 12, 2010, Haiti experienced its biggest earthquake in two centuries, with one third of its population seriously affected in the capital city of Port-au-Prince, with the infrastructure from roads, the port, hospitals, schools, etc., severely damaged, if not destroyed. Getting the humanitarian relief supplies from water, food, medicines, and associated services, to the victims has been a major challenge with the absence of well-planned and coordinated logistics a clear problem. There was no central and coordinated control of the distribution of relief supplies for weeks following this disaster (Cowell and Otterman (2010)). It is clear that a better-designed supply chain would have facilitated the relief efforts and would have resulted in less suffering and lives lost.

In this paper, we provide a system-optimization perspective for supply chain network design for critical needs that allows for the simultaneous determination of link capacities, through investments, and the product flows on various links, that is, the manufacturing, storage, distribution/shipment links, etc. At the same time, we allow for the flexibility associated with outsourcing. We believe that, in times of crises, a system-optimization 
approach is mandated since the demands for critical supplies should be met (as nearly as possible) at minimal total cost. The use of a profit maximization criterion, as in Nagurney (2010), may not appeal to stakeholders, whereas a cost minimization one demonstrates social responsibility and sensitivity. In addition, even in severe disasters and crises, there should be some information available, as to the expected demand for essential products, in different geographical locations, based on, for example, a previous census and/or governmental data collection.

A system-optimization perspective for supply chain network design for critical needs with outsourcing, as we demonstrate, captures, in a graphical manner, the options available, and provides flexibility in terms of the evaluation of trade-offs of producing "in-house" versus outsourcing. Importantly, we consider a generalized cost associated with the various supply chain activities, in order to subsume not only the financial cost but also the time element, any risk, etc. In particular, we capture in the model the uncertainty of the demand and the associated penalties for undersupply/shortages at various demand points.

System-optimization models have recently been developed for supply chain network integration in the case of mergers and acquisitions (see Nagurney (2009a), Nagurney and Woolley (2010), and Nagurney, Woolley, and Qiang (2009)). However, in those models, in contrast to the one in this paper, it is assumed that the capacities on the supply chain network links are fixed and known. It is worth noting that in 1967, there were 26 licensed manufacturers of vaccines in the US, whereas the number has now fallen to 6 (see Klein and Myers (2006)). Interestingly, the merging of manufacturers is one reason for the decreased number of manufacturers of vaccines.

An alternative approach (cf. Nagurney, Dong, and Zhang (2002), Zhang, Dong, and Nagurney (2003), Zhang (2006), Nagurney (2009b)) considers competition among decisionmakers in supply chains and uses equilibrium (as opposed to optimization) as the governing concept. In such supply chain network equilibrium models (see also Nagurney (2006) and the references therein) there are no explicit capacity link variables. The design issue in such models is, typically, handled by eliminating the links in the solution that have zero product flows. Moreover, in the case of critical needs, cost-minimization, rather than profitmaximization is the more appropriate objective function for decision-makers. An equilibrium 
approach to facility location which makes use of binary variables can be found in Miller, Friesz, and Tobin (1996).

It is worth noting that our perspective for supply chain network design in this paper is based, in part, on the concept of system-optimization in transportation and regional science (cf. Beckmann, McGuire, and Winsten (1956), Dafermos and Sparrow (1969), and Boyce, Mahmassani, and Nagurney (2005)). However, here we include also link capacity enhancements as decision variables, as well as explicit outsourcing, plus we also handle any existing capacities on the links. Moreover, here we also model uncertainty associated with the demand for the product at the demand points (see also Qiang, Nagurney, and Dong (2009) and the references therein).

This paper is organized as follows. In Section 2, we develop the supply chain network design model for critical needs with outsourcing in which capacity levels (associated with those links that the organization controls) and product flows are endogenous variables. We establish that the optimization problem is equivalent to a variational inequality problem, with nice features for computations. The solution of the supply chain network design model yields the optimal product flows and enhancement capacities on the supply chain network, and the optimal outsourced volumes, so that the total cost is minimized and the demands are satisfied as nearly as possible. We also discuss two distinct applications of our model to vaccine production and emergency preparedness and humanitarian logistics, and provide, for illustration purposes, several simple numerical examples. For completeness, in Section 3, we outline the algorithm which yields closed form expressions at each iteration for the product flows, the capacity enhancements, and the Lagrange multipliers. In Section 4 we compute solutions to additional supply chain network design numerical examples. In Section 5, we summarize the results in this paper and present our conclusions. 


\section{The Supply Chain Network Design Model for Critical Needs}

In this Section, we develop the supply chain network design model for critical needs. We assume that the organization (the government, corporation, humanitarian organization, etc.) responsible for ensuring that the demand for the essential product be met is considering the possible supply chain activities, associated with the product (be it medicine, vaccine, water, etc.), which are represented by a network. For clarity and definiteness, we consider the network topology depicted in Figure 1 but emphasize that the modeling framework developed here is not limited to such a network. Indeed, as will become apparent, what is required, to begin with, is the appropriate network topology with a top level (origin) node 1 corresponding to the organization and the bottom level (destination) nodes corresponding to the demand points that the organization must supply. We also allow for outsourcing of the product in terms of its production and delivery with the outsourcing links represented in Figure 1 as curved links. As we will show below, there will be a fixed cost associated with each outsourcing link to reflect the price for the product the organization has agreed to pay, if it decides to take that route.

The paths joining the origin node to the destination nodes represent sequences of supply chain network activities that ensure that the product is produced and, ultimately, delivered to those in need at the demand points. Hence, different supply chain network topologies to that depicted in Figure 1 correspond to distinct supply chain network problems. For example, if a product can be delivered directly to the demand points from a manufacturing plant, then there would be a direct link joining the corresponding nodes.

We assume that in the supply chain network topology there exists one path (or more) joining node 1 with each destination node. This assumption for the supply chain network design model guarantees that the demand at each demand point will be met as closely as possible, given that we also consider demand uncertainty, as discussed below. The solution of the model will then yield the optimal product flows and capacity investments at minimum total cost. Note that the supply chain network schematic, as in Figure 1, provides the foundation upon which the optimal supply chain network design will be determined.

In particular, as depicted in Figure 1, we assume that the organization is considering $n_{M}$ manufacturing facilities/plants; $n_{D}$ distribution centers, but must serve the $n_{R}$ demand 
points with respective demands given by: $d_{R_{1}}, d_{R_{2}}, \ldots, d_{R_{n_{R}}}$. The links from the toptiered node 1 are connected to the possible manufacturing nodes of the organization, which are denoted, respectively, by: $M_{1}, \ldots, M_{n_{M}}$, and these links represent the manufacturing links. The links from the manufacturing nodes, in turn, are connected to the possible distribution center nodes of the organization, and are denoted by $D_{1,1}, \ldots, D_{n_{D}, 1}$. These links correspond to the possible shipment links between the manufacturing plants and the distribution centers where the product will be stored. The links joining nodes $D_{1,1}, \ldots, D_{n_{D}, 1}$ with nodes $D_{1,2}, \ldots, D_{n_{D}, 2}$ correspond to the possible storage links. Finally, there are possible shipment links joining the nodes $D_{1,2}, \ldots, D_{n_{D}, 2}$ with the demand nodes: $R_{1}, \ldots, R_{n_{R}}$. As noted earlier, there are also outsourcing links, which join the top node to each bottom node. The organization does not control the capacities on these links since they have been established by the particular firm that corresponds to the outsource link prior.

We denote the supply chain network consisting of the graph $G=[N, L]$, where $N$ denotes the set of nodes and $L$ the set of links. Let $L^{1}$ and $L^{2}$ denote the links associated with "in house" supply chain activities and the outsourcing activities, respectively. Then $L \equiv L^{1} \cup L^{2}$. We further denote $n_{L^{1}}$ and $n_{L^{2}}$ as the number of links in the link set $L^{1}$ and $L^{2}$ respectively. Note that $G$ represents the topology of the full supply chain network possibilities (as in Figure 1, for example). The ultimate solution of the complete model will yield the optimal supply chain network design.

As mentioned in the Introduction, the formalism that we utilize is that of systemoptimization, where the organization wishes to determine which manufacturing plants it should operate and at what level; the same for the distribution centers, as well as how much of the product should be outsourced. In addition, the organization seeks to determine the capacity levels of the shipment links as well. We assume that the organization seeks to minimize the total costs associated with its production, storage, distribution activities, along with the total investment outlays to achieve the activity levels as given by the capacities on its various links, and the volumes of the product outsourced, subject to the demand being satisfied as nearly as possible at the demand points with associated penalties if the demands are not met. 


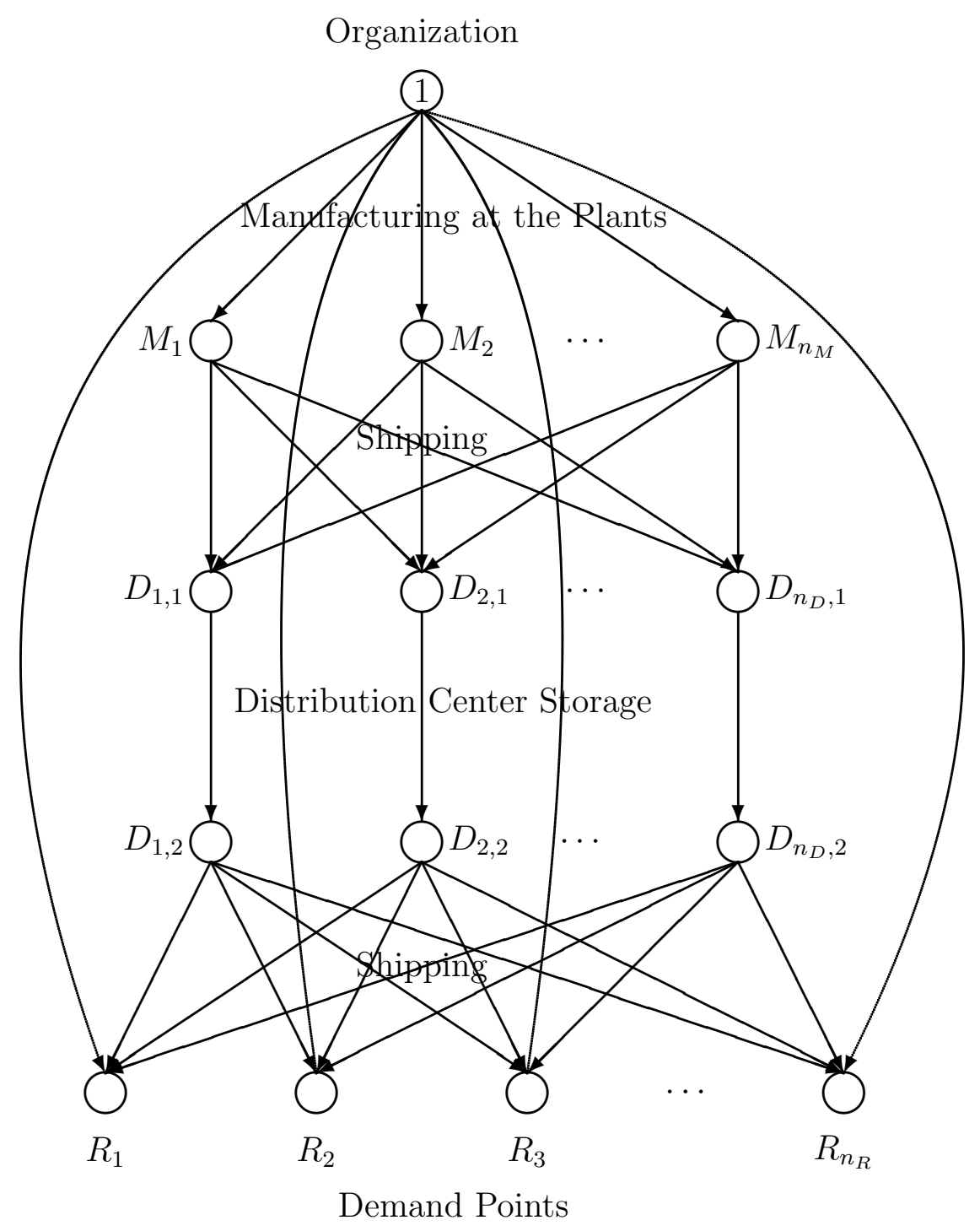

Figure 1: Supply Chain Network Topology with Outsourcing 
Associated with each link (cf. Figure 1) of the network is a total cost that reflects the total cost of operating the particular supply chain activity, that is, the manufacturing of the product, the shipment of the product, the storage of the product, etc., over the time horizon underlying the design problem. We denote, without any loss in generality, the links by $a, b$, etc., and the total cost on a link $a$ by $\hat{c}_{a}$. For the sake of generality, we note that the total costs are generalized costs and may include, for example, risk, time, etc. We also emphasize that the model is, in effect, not restricted to the topology in Figure 1.

A path $p$ in the network (see, e.g., Figure 1) joining node 1, which is the origin node, to a demand node, which is a destination node, represents the activities and their sequence associated with producing the product and having it, ultimately, delivered to those in need. Let $w_{k}$ denote the pair of origin/destination $(\mathrm{O} / \mathrm{D})$ nodes $\left(1, R_{k}\right)$ and let $P_{w_{k}}$ denote the set of paths, which represent alternative associated possible supply chain network processes, joining $\left(1, R_{k}\right)$. $P$ then denotes the set of all paths joining node 1 to the demand nodes. Let $n_{P}$ denote the number of paths from the organization to the demand markets.

Let $x_{p}$ represent the nonnegative flow of the product on path $p$ joining (origin) node 1 with a (destination) demand node that the organization is to supply with the critical product. Note that the paths corresponding to outsourcing consist of single links. In addition, these links are not contained in any other paths in the network.

For the convenience of expression, let

$$
v_{k} \equiv \sum_{p \in P_{w_{k}}} x_{p}, \quad k=1, \ldots, n_{R}
$$

where $v_{k}$ can be interpreted as the projected demand at demand market $k ; k=1, \ldots, n_{R}$.

We assume that the demand at each demand point is uncertain with a known probability distribution. Let $d_{k}$ denote the demand at demand point $k ; k=1, \ldots, n_{R}$, which is a random variable with probability density function given by $\mathcal{F}_{k}(t)$. Let $P_{k}$ be the probability distribution function of $d_{k}$, that is, $P_{k}\left(D_{k}\right)=P_{k}\left(d_{k} \leq D_{k}\right)=\int_{0}^{D_{k}} \mathcal{F}_{k}(t) d(t)$. Then,

$$
\begin{aligned}
& \Delta_{k}^{-} \equiv \max \left\{0, d_{k}-v_{k}\right\}, \quad k=1, \ldots, n_{R}, \\
& \Delta_{k}^{+} \equiv \max \left\{0, v_{k}-d_{k}\right\}, \quad k=1, \ldots, n_{R},
\end{aligned}
$$


where $\Delta_{k}^{-}$and $\Delta_{k}^{+}$represent the supply shortage and surplus at demand point $k$, respectively.

It has been argued by researchers that the demand in networks such as humanitarian aid supply chains is uncertain. At the same time, a relatively prompt response time may be required. It is, therefore, imperative for the cognizant organization to be fully prepared in terms of demand estimation in order to be able to fulfill the demand in a timely fashion (see, e.g., Altay (2008), Beamon and Kotleba (2006)).

The expected values of $\Delta_{k}^{-}$and $\Delta_{k}^{+}$are given by:

$$
\begin{aligned}
& E\left(\Delta_{k}^{-}\right)=\int_{v_{k}}^{\infty}\left(t-v_{k}\right) \mathcal{F}_{k}(t) d(t), \quad k=1, \ldots, n_{R}, \\
& E\left(\Delta_{k}^{+}\right)=\int_{0}^{v_{k}}\left(v_{k}-t\right) \mathcal{F}_{k}(t) d(t), \quad k=1, \ldots, n_{R} .
\end{aligned}
$$

We assume that the unit penalty of supply shortage at demand point $k$ is $\lambda_{k}^{-}$and that of supply surplus is $\lambda_{k}^{+}$. The expected total penalty at demand point $k ; k=1, \ldots, n_{R}$, is, hence,

$$
E\left(\lambda_{k}^{-} \Delta_{k}^{-}+\lambda_{k}^{+} \Delta_{k}^{+}\right)=\lambda_{k}^{-} E\left(\Delta_{k}^{-}\right)+\lambda_{k}^{+} E\left(\Delta_{k}^{+}\right)
$$

Note that $\lambda_{k}^{+}$denotes the unit penalty cost of having excessive supply at $k$, which includes the inventory cost, the cost of obsolescence for a perishable product, as well as the disposal cost, if relevant. $\lambda_{k}^{-}$, in turn, denotes the unit penalty cost of having a supply shortage at $k$, which corresponds to the social cost, associated with the loss of the well-being of the population, due to a shortage. Similar examples of penalty costs due to excessive supplies as well as to shortages, respectively, can be found in the literature (see, e.g., Dong, Zhang, and Nagurney (2004) and Nagurney and Matsypura (2005)). In the case of critical needs products such penalty costs are especially relevant since excessive supplies of critical needs products may lead to waste and environmental damage whereas shortages of critical needs products may lead to suffering and even death. These penalties can be assessed by the authority who is contracting with the organization to deliver the critical needs product.

In addition, we let $f_{a}$ denote the flow of the product on link $a$. Hence, we must have the following conservation of flow equations satisfied:

$$
f_{a}=\sum_{p \in P} x_{p} \delta_{a p}, \quad \forall a \in L,
$$


that is, the total amount of a product on a link is equal to the sum of the flows of the product on all paths that utilize that link.

Of course, we also have that the path flows must be nonnegative, that is,

$$
x_{p} \geq 0, \quad \forall p \in P
$$

since the product will be produced in nonnegative quantities.

We group the path flows, the link flows, and the projected demands into the respective vectors $x, f$, and $v$.

The total cost on a link, be it a manufacturing/production link, a shipment link, or a storage link is assumed to be a function of the flow of the product on the link; see, for example, Nagurney $(2006,2009 a)$ and the references therein. We have, thus, that

$$
\hat{c}_{a}=\hat{c}_{a}\left(f_{a}\right), \quad \forall a \in L
$$

The total cost on an outsource link is also of the form in (9a), with the proviso that we assume a fixed positive price $\rho_{a}$ charged for a unit of the product, which is negotiated between the outsource firm associated with that link and the organization. Hence, the total cost on an outsource link a takes the explicit form:

$$
\hat{c}_{a}=\rho_{a} f_{a}, \quad \forall a \in L^{2} .
$$

We assume that the total cost on each link is convex and is continuously differentiable.

We denote the nonnegative existing capacity on a link $a$ by $\bar{u}_{a}, \forall a \in L$. Note that the organization can add capacity to the "in house" link $a ; \forall a \in L^{1}$. We denote the total investment cost of adding capacity $u_{a}$ on link $a$ by $\hat{\pi}_{a}, \forall a \in L^{1}$, and assume that

$$
\hat{\pi}_{a}=\hat{\pi}_{a}\left(u_{a}\right), \quad \forall a \in L^{1},
$$

that is, the total cost associated with adding capacity $u_{a}$ on link $a$ is a function of the added capacity on the link. These functions are assumed to be convex and continuously differentiable. We group the added link capacities into the vector $u$. 
We note that the above assumptions on the underlying functions are not unreasonable since we are dealing with continuous variables and similar assumptions have been made for transportation network links as well as other supply chain links, including manufacturing links in the literature (see, e.g., Nagurney (2006)).

The supply chain network design optimization problem for critical needs faced by the organization can be expressed as follows. The organization seeks to determine the optimal levels of product processed on each supply chain network link (including the outsourcing links) coupled with the optimal levels of capacity investments in its supply chain network activities subject to the minimization of the total cost. The total cost includes the total cost of operating the various links, the total cost of capacity investments, and the expected total supply shortage/surplus penalty. Hence, the organization must solve the following problem:

$$
\text { Minimize } \sum_{a \in L} \hat{c}_{a}\left(f_{a}\right)+\sum_{a \in L^{1}} \hat{\pi}_{a}\left(u_{a}\right)+\sum_{k=1}^{n_{R}}\left(\lambda_{k}^{-} E\left(\Delta_{k}^{-}\right)+\lambda_{k}^{+} E\left(\Delta_{k}^{+}\right)\right)
$$

subject to: constraints (1), (7), (8), and

$$
\begin{gathered}
f_{a} \leq \bar{u}_{a}+u_{a}, \quad \forall a \in L^{1}, \\
f_{a} \leq \bar{u}_{a}, \quad \forall a \in L^{2}, \\
0 \leq u_{a}, \quad \forall a \in L^{1} .
\end{gathered}
$$

Constraint (13) reflects that the outsource firms cannot produce/deliver more of the product than their existing capacity on the corresponding link. Constraint (14) indicates that the existing capacities are not allowed to be reduced in this problem, but can be increased or remain unchanged. Constraint (12), in turn, guarantees that the product flow on a link does not exceed that link's capacity.

The first variational inequality that we will present below is in path flows (rather than in link flows) since such a formulation will enable for a very elegant and simple computation of solutions. Towards that end, in view of (1) through (5), we note that $\lambda_{k}^{-} E\left(\Delta_{k}^{-}\right)+\lambda_{k}^{+} E\left(\Delta_{k}^{+}\right)$ is a function of the path flow vector $x$. Objective function (11), in light of the above, and in lieu of (7), can, hence, be expressed solely in terms of the path flow variables and the 
capacity enhancement variables, and the same holds true for the constraints (12) through (14) with the proviso that we also retain (8).

We now present some additional preliminaries. For each O/D pair $w_{k}$,

$$
\begin{gathered}
\frac{\partial E\left(\Delta_{k}^{-}\right)}{\partial x_{p}}=\frac{\partial E\left(\Delta_{k}^{-}\right)}{\partial v_{k}} \cdot \frac{\partial v_{k}}{\partial x_{p}}=P_{k}\left(\sum_{p \in P_{w_{k}}} x_{p}\right)-1, \quad \forall p \in P_{w_{k}}, \\
\frac{\partial E\left(\Delta_{k}^{+}\right)}{\partial x_{p}}=\frac{\partial E\left(\Delta_{k}^{+}\right)}{\partial v_{k}} \cdot \frac{\partial v_{k}}{\partial x_{p}}=P_{k}\left(\sum_{p \in P_{w_{k}}} x_{p}\right), \quad \forall p \in P_{w_{k}},
\end{gathered}
$$

and

$$
\frac{\partial^{2}}{\partial x_{p}^{2}}\left[\lambda_{k}^{-} E\left(\Delta_{k}^{-}\right)+\lambda_{k}^{+} E\left(\Delta_{k}^{+}\right)\right]=\left(\lambda_{k}^{-}+\lambda_{k}^{+}\right) \mathcal{F}_{k}\left(\sum_{p \in P_{w_{k}}} x_{p}\right), \quad \forall p \in P_{w_{k}} .
$$

Hence, it is obvious that $\lambda_{k}^{-} E\left(\Delta_{k}^{-}\right)+\lambda_{k}^{+} E\left(\Delta_{k}^{+}\right)$is convex.

Clearly, the solution of the above optimization problem will yield the product flows and the link capacities that minimize the total costs associated with the supply chain network design faced by the organization. Under the above imposed assumptions, the optimization problem is a convex optimization problem.

We associate the Lagrange multiplier $\omega_{a}$ with constraint (12) for link $a \in L^{1}$ and we denote the associated optimal Lagrange multiplier by $\omega_{a}^{*}$. Similarly, Lagrange multiplier $\gamma_{a}$ is associated with constraint (13) for link $a \in L^{2}$ with the optimal multiplier denoted by $\gamma_{a}^{*}$. These two terms may also be interpreted as the price or value of an additional unit of capacity on link $a$. We group these Lagrange multipliers into the vectors $\omega$ and $\gamma$, respectively.

Let $K$ denote the feasible set such that

$$
K \equiv\left\{(x, u, \omega, \gamma) \mid x \in R_{+}^{n_{P}}, u \in R_{+}^{n_{L^{1}}}, \omega \in R_{+}^{n_{L^{1}}}, \text { and } \gamma \in R_{+}^{n_{L^{2}}}\right\}
$$

We now state the following result in which we provide variational inequality formulations of the problem in both path flows and in link flows, respectively. 


\section{Theorem 1}

The optimization problem (11), subject to the constraints above, is equivalent to the variational inequality problem: determine the vector of optimal path flows, the vector of optimal link capacity enhancements, and the vectors of optimal Lagrange multipliers $\left(x^{*}, u^{*}, \omega^{*}, \gamma^{*}\right) \in$ $K$, such that:

$$
\begin{gathered}
\sum_{k=1}^{n_{R}} \sum_{p \in P_{w_{k}}}\left[\frac{\partial \hat{C}_{p}\left(x^{*}\right)}{\partial x_{p}}+\sum_{a \in L^{1}} \omega_{a}^{*} \delta_{a p}+\sum_{a \in L^{2}} \gamma_{a}^{*} \delta_{a p}+\lambda_{k}^{+} P_{k}\left(\sum_{p \in P_{w_{k}}} x_{p}^{*}\right)-\lambda_{k}^{-}\left(1-P_{k}\left(\sum_{p \in P_{w_{k}}} x_{p}^{*}\right)\right]\right. \\
\times\left[x_{p}-x_{p}^{*}\right] \\
+\sum_{a \in L^{1}}\left[\frac{\partial \hat{\pi}_{a}\left(u_{a}^{*}\right)}{\partial u_{a}}-\omega_{a}^{*}\right] \times\left[u_{a}-u_{a}^{*}\right]+\sum_{a \in L^{1}}\left[\bar{u}_{a}+u_{a}^{*}-\sum_{p \in P} x_{p}^{*} \delta_{a p}\right] \times\left[\omega_{a}-\omega_{a}^{*}\right] \\
+\sum_{a \in L^{2}}\left[\bar{u}_{a}-\sum_{p \in P} x_{p}^{*} \delta_{a p}\right] \times\left[\gamma_{a}-\gamma_{a}^{*}\right] \geq 0, \quad \forall(x, u, \omega, \gamma) \in K,
\end{gathered}
$$

where $\frac{\partial \hat{C}_{p}(x)}{\partial x_{p}} \equiv \sum_{a \in L} \frac{\partial \hat{c}_{a}\left(f_{a}\right)}{\partial f_{a}} \delta_{a p}$ for paths $p \in P_{w_{k}} ; k=1, \ldots, n_{R}$.

In addition, (18) can be reexpressed in terms of links flows as: determine the vector of optimal link flows, the vectors of optimal projected demands and link capacity enhancements, and the vectors of optimal Lagrange multipliers $\left(f^{*}, v^{*}, u^{*}, \omega^{*}, \gamma^{*}\right) \in K^{1}$, such that:

$$
\begin{gathered}
\sum_{a \in L^{1}}\left[\frac{\partial \hat{c}_{a}\left(f_{a}^{*}\right)}{\partial f_{a}}+\omega_{a}^{*}\right] \times\left[f_{a}-f_{a}^{*}\right] \\
+\sum_{a \in L^{2}}\left[\frac{\partial \hat{c}_{a}\left(f_{a}^{*}\right)}{\partial f_{a}}+\gamma_{a}^{*}\right] \times\left[f_{a}-f_{a}^{*}\right]+\sum_{a \in L^{1}}\left[\frac{\partial \hat{\pi}_{a}\left(u_{a}^{*}\right)}{\partial u_{a}}-\omega_{a}^{*}\right] \times\left[u_{a}-u_{a}^{*}\right] \\
+\sum_{k=1}^{n_{R}}\left[\lambda_{k}^{+} P_{k}\left(v_{k}^{*}\right)-\lambda_{k}^{-}\left(1-P_{k}\left(v_{k}^{*}\right)\right)\right] \times\left[v_{k}-v_{k}^{*}\right] \\
+\sum_{a \in L^{1}}\left[\bar{u}_{a}+u_{a}^{*}-f_{a}^{*}\right] \times\left[\omega_{a}-\omega_{a}^{*}\right] \\
+\sum_{a \in L^{2}}\left[\bar{u}_{a}-f_{a}^{*}\right] \times\left[\gamma_{a}-\gamma_{a}^{*}\right] \geq 0, \quad \forall(f, v, u, \omega, \gamma) \in K^{1}
\end{gathered}
$$

where $K^{1} \equiv\{(f, v, u, \omega, \gamma) \mid \exists x \geq 0$, and (1), (7), (8), and (14) hold, and $\omega \geq 0, \gamma \geq 0\}$.

Proof: See Bertsekas and Tsitsiklis (1989) page 287. 
Note that both variational inequalities (18) and (19) can be put into standard form (see Nagurney (1993)): determine $X^{*} \in \mathcal{K}$ such that:

$$
\left\langle F\left(X^{*}\right)^{T}, X-X^{*}\right\rangle \geq 0, \quad \forall X \in \mathcal{K}
$$

where $\langle\cdot, \cdot\rangle$ denotes the inner product in $n$-dimensional Euclidean space. We utilize the path flow formulation (18) since it yields very simple expressions for algorithmic computations; hence, we now put (18) into standard form (20). We define the column vectors: $X \equiv$ $(x, u, \omega, \gamma)$ and $F(X) \equiv\left(F_{1}(X), F_{2}(X), F_{3}(X), F_{4}(X)\right)$, such that

$$
\begin{gathered}
F_{1}(X)=\left[\frac{\partial \hat{C}_{p}(x)}{\partial x_{p}}+\sum_{a \in L^{1}} \omega_{a} \delta_{a p}+\sum_{a \in L^{2}} \gamma_{a} \delta_{a p}+\lambda_{k}^{+} P_{k}\left(\sum_{p \in P_{w_{k}}} x_{p}\right)-\lambda_{k}^{-}\left(1-P_{k}\left(\sum_{p \in P_{w_{k}}} x_{p}\right)\right)\right. \\
\left.p \in P_{w_{k}} ; k=1, \ldots, n_{R}\right], \quad F_{2}(X)=\left[\frac{\partial \hat{\pi}_{a}\left(u_{a}\right)}{\partial u_{a}}-\omega_{a} ; a \in L^{1}\right], \\
F_{3}(X)=\left[\bar{u}_{a}+u_{a}-\sum_{p \in P} x_{p} \delta_{a p} ; \quad a \in L^{1}\right], \quad F_{4}(X)=\left[\bar{u}_{a}-\sum_{p \in P} x_{p} \delta_{a p} ; \quad a \in L^{2}\right] .
\end{gathered}
$$

Also, we define $\mathcal{K} \equiv K$. Then (18) can be reexpressed as (20).

Specifically, variational inequality (18) can be easily solved using the Euler method, which is induced by the general iterative scheme of Dupuis and Nagurney (1993) and which we fully discuss in Section 3. In particular, because of the variational inequality formulation (18), the Euler method will yield, at each iteration, closed form expressions for the path flows, the link capacity enhancements, and the Lagrange multipliers.

Once we have solved problem (18) we can obtain the solution $\left(f^{*}, u^{*}\right)$ (by using (7), which relates the link flows to the path flows) that minimizes the total cost (cf. (11)) associated with the design of the supply chain network for critical needs.

\subsection{Applications to Vaccine Production and Emergency Preparedness and Hu- manitarian Logistics}

We now discuss two specific applications of the above model. The first application is to the production of $\mathrm{H} 1 \mathrm{~N} 1$ vaccine and the second application is to a specific humanitarian logistics operation which also illustrates the importance of emergency preparedness. 
Consider a vaccine manufacturer such as Sanofi-Aventis who is gearing up for next year's production of H1N1 (swine) flu vaccine. Since it already has been involved in such a vaccine production this year it has some existing capacity. Governments around the world are beginning to contract with this company for next year's flu vaccine. By applying the general theoretical model to the company's data, which would also include the projected demand forecasts based on the needs of the populations that it is to serve (and based on information provided by the contracting governments), the firm can determine whether it needs to expand its facilities (or not), how much of the vaccine to produce where, how much to store where, and how much to have shipped to the various demand points. Also, it can determine whether it should outsource any of its vaccine production and at what level. The solution of the model yields the minimal total cost with the inclusion of penalties, which we can expect, in this application, to be higher for underproduction and lower for overproduction. These penalties, in this case, can be assessed also by the contracting governments. The firm by solving the model with its company-relevant data can then ensure that the price that it receives for its vaccine production and delivery is appropriate and that it recovers its incurred costs and obtains, if negotiated correctly, an equitable profit.

Another application is to emergency preparedness and humanitarian logistics. In August 2005 Hurricane Katrina hit the US and this natural disaster cost immense damage with repercussions that continue to this day. Wal-Mart, the global corporation, was at the forefront of hurricane relief (see Barbara and Gillis (2005)). While US state and federal officials came under severe criticism for their handling of the storm's aftermath, Wal-Mart had prepared in advance and through its logistical efficiencies had dozens of trucks loaded with supplies for delivery before the hurricane even hit landfall. It was able to deliver much needed supplies in the form of food and water to the needy citizens and took advantage of its major distribution center which is located in the state of Mississippi.

A company can, using the above model, prepare and plan for an emergency such as a natural disaster in the form of a hurricane and identify where to store a necessary product (such as food packets, for example) so that the items can be delivered to the demand points in a timely manner and at minimal total cost. Note that, in the case of Wal-Mart and Hurricane Katrina, Wal-Mart had existing capacity in the form of its large distribution center and numerous stores in the southern region of the US where Hurricane Katrina had 
its greatest physical impact. Hence, it was able to distribute needed supplies and, in addition, achieved an enormous amount of goodwill.

\subsection{Simple Supply Chain Network Design Numerical Examples}

In order to further illustrate the above model, we now present several simple examples.

Consider the supply chain network topology in Figure 2 in which the organization is considering a single manufacturing plant, a single distribution center for storing the critical need product and is to serve a single demand point. The links are labeled as in Figure 2, that is, $a, b, c, d$, and $e$, with $e$ denoting the outsourcing link.

Below we provide solutions to four supply chain network design examples, all of which consider the initial topology given in Figure 2.

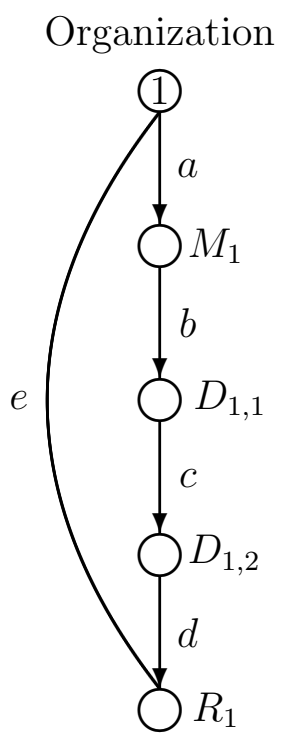

Demand Point

Figure 2: Simple Network for the Numerical Examples 


\section{Example 1}

The total cost functions on the links were:

$$
\begin{gathered}
\hat{c}_{a}\left(f_{a}\right)=.5 f_{a}^{2}+f_{a}, \quad \hat{c}_{b}\left(f_{b}\right)=.5 f_{b}^{2}+2 f_{b}, \quad c_{c}\left(f_{c}\right)=.5 f_{c}^{2}+f_{c}, \quad \hat{c}_{d}\left(f_{d}\right)=.5 f_{d}^{2}+2 f_{d} \\
\hat{c}_{e}\left(f_{e}\right)=5 f_{e} .
\end{gathered}
$$

The investment capacity cost functions were: $\hat{\pi}_{a}\left(u_{a}\right)=.5 u_{a}^{2}+u_{a}, \quad \forall a \in L^{1}$.

The existing capacities were: $\bar{u}_{a}=0, \quad \forall a \in L^{1}$, and $\bar{u}_{e}=2$.

The paths were defined as: $p_{1}=(a, b, c, d)$ and $p_{2}=e$ with $\mathrm{O} / \mathrm{D}$ pair $w_{1}=\left(1, R_{1}\right)$.

We assumed that the demand for the product followed a uniform distribution on the interval $[0,10]$ so that: $P_{1}\left(\sum_{p \in P_{w_{1}}} x_{p}\right)=\frac{\sum_{p \in P_{1}} x_{p}}{10}$.

The penalties were: $\lambda_{1}^{-}=10, \quad \lambda_{1}^{+}=0$.

The path flow solution was: $x_{p_{1}}^{*}=0.00, x_{p_{2}}^{*}=2.00$, which corresponds to the link flow pattern: $f_{a}^{*}=f_{b}^{*}=f_{c}^{*}=f_{d}^{*}=0.00, f_{e}^{*}=2.00$.

The capacity investments were: $u_{a}^{*}=0.00, \quad \forall a \in L^{1}$.

The optimal Lagrange multipliers were: $\omega_{a}^{*}=1.00, \quad \forall a \in L^{1}, \gamma_{e}^{*}=3.00$.

Hence, the critical product was obtained and delivered to the demand point exclusively through outsourcing.

It is easy to verify that, indeed, the above solution satisfies variational inequality (18). Since the current capacities in the "in-house" supply chain links are zero, it is more costly to expand them than to outsource. Consequently, the organization chooses to outsource the product for production and delivery. The optimal supply chain network design for Example 1, hence, corresponds to the network in Figure 2 but with the only remaining link being link $e$ since the capacities (and flows) on all other links are zero at the optimal solution. 


\section{Example 2}

Example 2 had the same data as Example 1 except that we now increased the penalty associated with product shortage from 10 to 50 , that is, we now set $\lambda_{1}^{-}=50$.

The new solution was as follows. The path flow solution was now: $x_{p_{1}}^{*}=2.31, \quad x_{p_{2}}^{*}=$ 2.00, which corresponds to the link flow pattern: $f_{a}^{*}=f_{b}^{*}=f_{c}^{*}=f_{d}^{*}=2.31, f_{e}^{*}=2.00$.

The capacity investments were: $u_{a}^{*}=2.31, \quad \forall a \in L^{1}$.

The optimal Lagrange multipliers were: $\omega_{a}^{*}=3.31, \quad \forall a \in L^{1}, \gamma_{e}^{*}=23.46$.

Hence, the critical product was now manufactured and distributed by the organization and also outsourced.

Since the penalty cost for under-supplying is increased, the organization increased its "in-house" capacity and product output. The optimal supply chain network design is as in Figure 2 since now all links have positive capacities and flows.

\section{Example 3}

Example 3 had the same data as Example 2 except that $\bar{u}_{a}=3$ for all the links $a \in L^{1}$. This means that the organization does not have to construct its supply chain activities from scratch as in Examples 1 and 2 but does have some existing capacity.

The new path flow solution was: $x_{p_{1}}^{*}=3.23, \quad x_{p_{2}}^{*}=2.00$, which corresponds to the link flow pattern: $f_{a}^{*}=f_{b}^{*}=f_{c}^{*}=f_{d}^{*}=3.23, f_{e}^{*}=2.00$.

The capacity investments were: $u_{a}^{*}=0.23, \quad \forall a \in L^{1}$.

The optimal Lagrange multipliers were: $\omega_{a}^{*}=1.23, \quad \forall a \in L^{1}, \gamma_{e}^{*}=18.84$.

Given the existing capacities in the "in-house" supply chain links, the organization chooses to supply more critical product from the local manufacturers and distributors. The optimal supply chain network design remains as in Figure 2 since now all links have positive capacities and flows. 


\section{Example 4}

Example 4 had the total cost functions on the links given by:

$$
\hat{c}_{a}\left(f_{a}\right)=f_{a}^{2}, \quad \hat{c}_{b}\left(f_{b}\right)=f_{b}^{2}, \quad c_{c}\left(f_{c}\right)=f_{c}^{2}, \quad \hat{c}_{d}\left(f_{d}\right)=f_{d}^{2}, \quad \hat{c}_{e}\left(f_{e}\right)=100 f_{e} .
$$

The investment capacity cost functions were: $\hat{\pi}_{a}\left(u_{a}\right)=u_{a}^{2}, \quad \forall a \in L^{1}$.

The existing capacities were: $\bar{u}_{a}=10, \quad \forall a \in L$.

The paths were defined, as in the previous examples, that is: $p_{1}=(a, b, c, d)$ and $p_{2}=e$.

We assumed that the demand followed a uniform distribution on the interval $[10,20]$ so that

$$
P_{1}\left(\sum_{p \in P_{w_{1}}} x_{p}\right)=\frac{\sum_{p \in P_{w_{1}}} x_{p}-10}{10} .
$$

The penalties were: $\lambda_{1}^{-}=1000, \quad \lambda_{1}^{+}=10$.

The path flow solution was: $x_{p_{1}}^{*}=11.25, x_{p_{2}}^{*}=7.66$, which corresponds to the link flow pattern: $f_{a}^{*}=f_{b}^{*}=f_{c}^{*}=f_{d}^{*}=11.25, f_{e}^{*}=7.66$.

The capacity investments were: $u_{a}^{*}=1.25, \quad \forall a \in L^{1}$.

The optimal Lagrange multipliers were: $\omega_{a}^{*}=2.50, \quad \forall a \in L^{1}, \gamma_{e}^{*}=0.00$.

In this example, since the penalty cost for under-supplying is much higher than that of over-supplying, the organization needs to both expand the "in-house" capacities and to outsource the production and delivery of the product to the demand point.

\section{The Algorithm}

In this Section, we recall the Euler method, which is induced by the general iterative scheme of Dupuis and Nagurney (1993). Its realization for the solution of supply chain network design problems governed by variational inequality (18) (and (20)) yields subproblems that can be solved explicitly and in closed form.

Specifically, recall that at an iteration $\tau$ of the Euler method (see also Nagurney and 
Zhang (1996)) one computes:

$$
X^{\tau+1}=P_{\mathcal{K}}\left(X^{\tau}-a_{\tau} F\left(X^{\tau}\right)\right),
$$

where $P_{\mathcal{K}}$ is the projection on the feasible set $\mathcal{K}$ and $F$ is the function that enters the variational inequality problem: determine $X^{*} \in \mathcal{K}$ such that

$$
\left\langle F\left(X^{*}\right)^{T}, X-X^{*}\right\rangle \geq 0, \quad \forall X \in \mathcal{K},
$$

where $\langle\cdot, \cdot\rangle$ is the inner product in $n$-dimensional Euclidean space, $X \in R^{n}$, and $F(X)$ is an $n$-dimensional function from $\mathcal{K}$ to $R^{n}$, with $F(X)$ being continuous (see also (20)).

As shown in Dupuis and Nagurney (1993); see also Nagurney and Zhang (1996), for convergence of the general iterative scheme, which induces the Euler method, among other methods, the sequence $\left\{a_{\tau}\right\}$ must satisfy: $\sum_{\tau=0}^{\infty} a_{\tau}=\infty, a_{\tau}>0, a_{\tau} \rightarrow 0$, as $\tau \rightarrow \infty$. Specific conditions for convergence of this scheme can be found for a variety of network-based problems, similar to those constructed here, in Nagurney and Zhang (1996) and the references therein. Applications to the solution of network oligopolies and spatial network equilibria can be found, respectively, in Nagurney, Dupuis, and Zhang (1994) and in Nagurney, Takayama, and Zhang (1995). We also note the collection of interesting papers with both models and algorithms for a variety of network-based problems, including dynamic ones, in the volume edited by Friesz (2007).

\section{Explicit Formulae for the Euler Method Applied to the Supply Chain Network Design Variational Inequality (18)}

The elegance of this procedure for the computation of solutions to the supply chain network design problem modeled in Section 2 can be seen in the following explicit formulae. Indeed, (21) for the supply chain design network problem governed by variational inequality problem (18) yields the following closed form expressions for the product path flows, the capacity enhancement capacities, and the Lagrange multipliers, respectively:

$$
\begin{aligned}
x_{p}^{\tau+1}= & \max \left\{0, x_{p}^{\tau}+a_{\tau}\left(\lambda_{k}^{-}\left(1-P_{k}\left(\sum_{p \in P_{w_{k}}} x_{p}^{\tau}\right)\right)-\lambda_{k}^{+} P_{k}\left(\sum_{p \in P_{w_{k}}} x_{p}^{\tau}\right)\right.\right. \\
& \left.\left.-\frac{\partial \hat{C}_{p}\left(x^{\tau}\right)}{\partial x_{p}}-\sum_{a \in L^{1}} \omega_{a}^{\tau} \delta_{a p}-\sum_{a \in L^{2}} \gamma_{a}^{\tau} \delta_{a p}\right)\right\}, \forall p \in P ;
\end{aligned}
$$




$$
\begin{array}{cc}
u_{a}^{\tau+1}=\max \left\{0, u_{a}^{\tau}+a_{\tau}\left(\omega_{a}^{\tau}-\frac{\partial \hat{\pi}_{a}\left(u_{a}^{\tau}\right)}{\partial u_{a}}\right)\right\}, & \forall a \in L^{1} ; \\
\omega_{a}^{\tau+1}=\max \left\{0, \omega_{a}^{\tau}+a_{\tau}\left(\sum_{p \in P} x_{p}^{\tau} \delta_{a p}-\bar{u}_{a}-u_{a}^{\tau}\right)\right\}, \quad \forall a \in L^{1} . \\
\gamma_{a}^{\tau+1}=\max \left\{0, \gamma_{a}^{\tau}+a_{\tau}\left(\sum_{p \in P} x_{p}^{\tau} \delta_{a p}-\bar{u}_{a}\right)\right\}, \quad \forall a \in L^{2} .
\end{array}
$$

In the next Section, we solve additional supply chain network design problems using the above algorithmic scheme.

\section{Additional Numerical Examples}

The Euler method for the solution of variational inequalities (18) was implemented in FORTRAN. A Unix system at the University of Massachusetts Amherst was used for all the computations below. We set the sequence $a_{\tau}=.1\left(1, \frac{1}{2}, \frac{1}{2}, \ldots\right)$. The convergence tolerance was $\epsilon=10^{-5}$. We initialized the algorithm by setting the projected demand to 100 at each demand point and by equally distributing it among the paths joining node 1 to each demand point. All other variables were initialized to zero.

The supply chain network topology for all the examples in this Section is as depicted in Figure 3 with the links defined by numbers as in Figure 3. The numerical examples, hence, consisted of an organization faced with 3 possible manufacturing plants, 2 distribution centers, and had to supply the 3 demand points. Also, as in Figure 3, we assumed that there were 3 outsourcing possibilities, with each such firm (or firms) serving a specific demand point.

The data for the specific examples along with the solutions are reported in the corresponding tables below. The solutions are reported in link form due to the number of paths. 


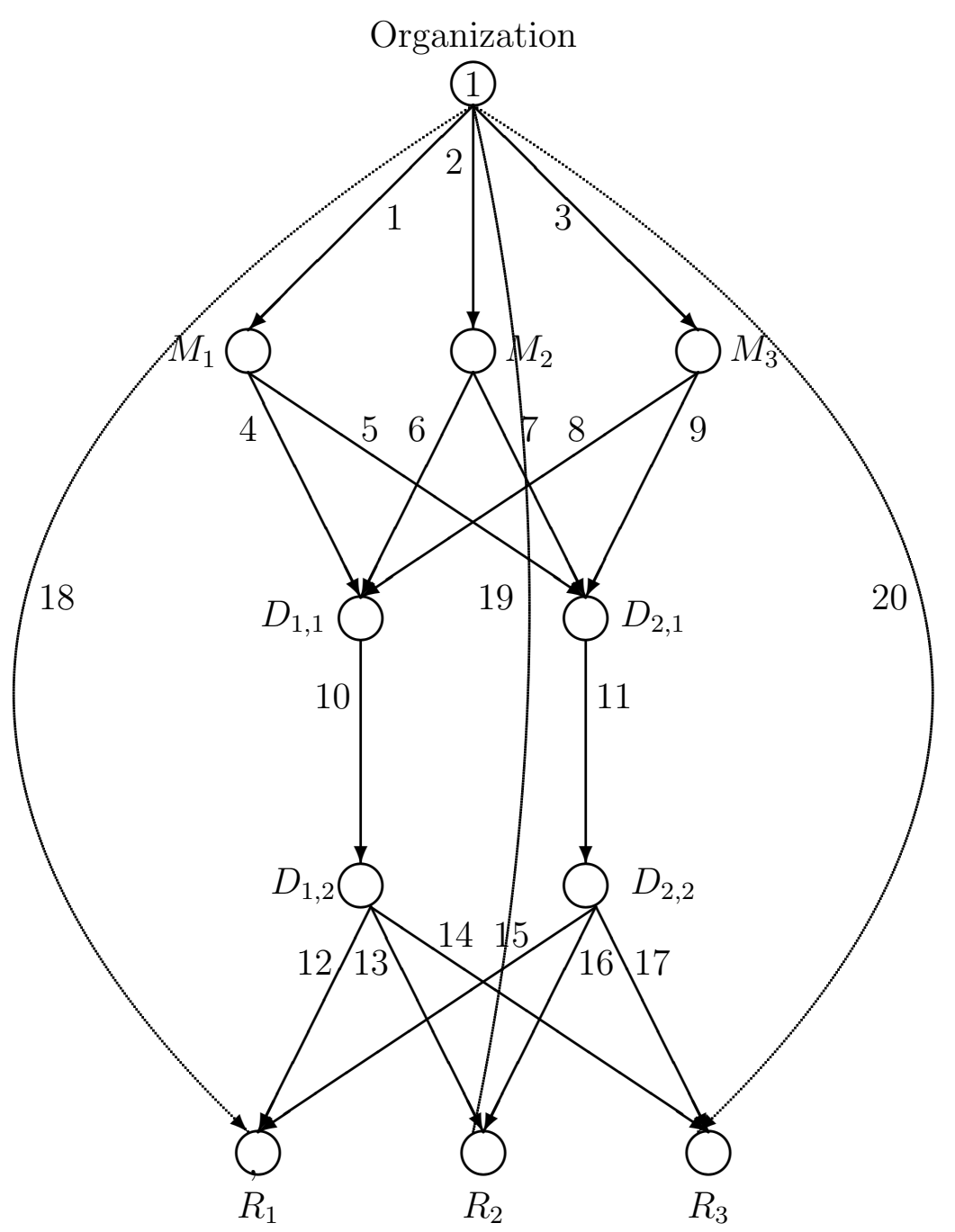

Figure 3: The Supply Chain Network Topology $G=[N, L]$ for the Examples in Section 4 


\section{Example 5}

We assumed that the demands at the three demand points followed a uniform probability distribution function on the intervals $[0,10],[0,20]$, and $[0,30]$, respectively, so that

$$
\begin{aligned}
& P_{1}\left(\sum_{p \in P_{w_{1}}} x_{p}\right)=\frac{\sum_{p \in P_{w_{1}}} x_{p}}{10}, \\
& P_{2}\left(\sum_{p \in P_{w_{2}}} x_{p}\right)=\frac{\sum_{p \in P_{w_{2}}} x_{p}}{20}, \\
& P_{3}\left(\sum_{p \in P_{w_{3}}} x_{p}\right)=\frac{\sum_{p \in P_{w_{3}}} x_{p}}{30},
\end{aligned}
$$

where $w_{1}=\left(1, R_{1}\right), w_{2}=\left(1, R_{2}\right)$, and $w_{3}=\left(1, R_{3}\right)$.

The penalties were:

$$
\begin{array}{ll}
\lambda_{1}^{-}=50, & \lambda_{1}^{+}=0, \\
\lambda_{2}^{-}=50, & \lambda_{2}^{+}=0, \\
\lambda_{3}^{-}=50, & \lambda_{3}^{+}=0 .
\end{array}
$$

The capacities associated with the three outsourcing links were:

$$
\bar{u}_{18}=5, \quad \bar{u}_{19}=10, \quad \bar{u}_{20}=5 .
$$

We set $\bar{u}_{a}=0$ for all links $a \in L^{1}$, that is, the organization begins its supply chain network activities from "scratch" and is assumed in this example to have no existing capacities.

The total cost functions were as reported in Table 1 where we also provide the computed solution using the Euler method.

Note that the optimal supply chain network design for Example 5 is, hence, as in Figure 3 but with links 13, 15, and 16 removed since those links have zero capacities and associated flows. Note that the organization took advantage of outsourcing to the full capacity available. 
Table 1: Total Cost Functions and Solution for Example 5

\begin{tabular}{|c|c|c|c|c|c|c|}
\hline Link $a$ & $\hat{c}_{a}\left(f_{a}\right)$ & $\hat{\pi}_{a}\left(u_{a}\right)$ & $f_{a}^{*}$ & $u_{a}^{*}$ & $\omega_{a}^{*}$ & $\gamma_{a}^{*}$ \\
\hline 1 & $f_{1}^{2}+2 f_{1}$ & $.5 u_{1}^{2}+u_{1}$ & 1.34 & 1.34 & 2.34 & - \\
\hline 2 & $.5 f_{2}^{2}+f_{2}$ & $.5 u_{2}^{2}+u_{2}$ & 2.47 & 2.47 & 3.47 & - \\
\hline 3 & $.5 f_{3}^{2}+f_{3}$ & $.5 u_{3}^{2}+u_{3}$ & 2.05 & 2.05 & 3.05 & - \\
\hline 4 & $1.5 f_{4}^{2}+2 f_{4}$ & $.5 u_{4}^{2}+u_{4}$ & 0.61 & 0.61 & 1.61 & - \\
\hline 5 & $f_{5}^{2}+3 f_{5}$ & $.5 u_{5}^{2}+u_{5}$ & 0.73 & 0.73 & 1.73 & - \\
\hline 6 & $f_{6}^{2}+2 f_{6}$ & $.5 u_{6}^{2}+u_{6}$ & 0.83 & 0.83 & 1.83 & - \\
\hline 7 & $.5 f_{7}^{2}+2 f_{7}$ & $.5 u_{7}^{2}+u_{7}$ & 1.64 & 1.64 & 2.64 & - \\
\hline 8 & $.5 f_{8}^{2}+2 f_{8}$ & $.5 u_{8}^{2}+u_{8}$ & 1.67 & 1.67 & 2.67 & - \\
\hline 9 & $f_{9}^{2}+5 f_{9}$ & $.5 u_{9}^{2}+u_{9}$ & 0.37 & 0.37 & 1.37 & - \\
\hline 10 & $.5 f_{10}^{2}+2 f_{10}$ & $.5 u_{10}^{2}+u_{10}$ & 3.11 & 3.11 & 4.11 & - \\
\hline 11 & $f_{11}^{2}+f_{11}$ & $.5 u_{11}^{2}+u_{11}$ & 2.75 & 2.75 & 3.75 & - \\
\hline 12 & $.5 f_{12}^{2}+2 f_{12}$ & $.5 u_{12}^{2}+u_{12}$ & 0.04 & 0.04 & 1.04 & - \\
\hline 13 & $.5 f_{13}^{2}+5 f_{13}$ & $.5 u_{13}^{2}+u_{13}$ & 0.00 & 0.00 & 0.45 & - \\
\hline 14 & $f_{14}^{2}$ & $.5 u_{14}^{2}+u_{14}$ & 3.07 & 3.07 & 4.07 & - \\
\hline 15 & $f_{15}^{2}+2 f_{15}$ & $.5 u_{15}^{2}+u_{15}$ & 0.00 & 0.00 & 0.45 & - \\
\hline 16 & $.5 f_{16}^{2}+3 f_{16}$ & $.5 u_{16}^{2}+u_{16}$ & 0.00 & 0.00 & 0.45 & - \\
\hline 17 & $.5 f_{17}^{2}+2 f_{17}$ & $.5 u_{17}^{2}+u_{17}$ & 2.75 & 2.75 & 3.75 & - \\
\hline 18 & $10 f_{18}$ & - & 5.00 & - & - & 14.77 \\
\hline 19 & $12 f_{19}$ & - & 10.00 & - & - & 13.00 \\
\hline 20 & $15 f_{20}$ & - & 5.00 & - & - & 16.96 \\
\hline
\end{tabular}


Table 2: Total Cost Functions and Solution for Example 6

\begin{tabular}{|c|c|c|c|c|c|c|}
\hline Link $a$ & $\hat{c}_{a}\left(f_{a}\right)$ & $\hat{\pi}_{a}\left(u_{a}\right)$ & $f_{a}^{*}$ & $u_{a}^{*}$ & $\omega_{a}^{*}$ & $\gamma_{a}^{*}$ \\
\hline 1 & $f_{1}^{2}+2 f_{1}$ & $.5 u_{1}^{2}+u_{1}$ & 1.84 & 0.00 & 0.00 & - \\
\hline 2 & $.5 f_{2}^{2}+f_{2}$ & $.5 u_{2}^{2}+u_{2}$ & 4.51 & 0.00 & 0.00 & - \\
\hline 3 & $.5 f_{3}^{2}+f_{3}$ & $.5 u_{3}^{2}+u_{3}$ & 3.85 & 0.00 & 0.00 & - \\
\hline 4 & $1.5 f_{4}^{2}+2 f_{4}$ & $.5 u_{4}^{2}+u_{4}$ & 0.88 & 0.00 & 0.00 & - \\
\hline 5 & $f_{5}^{2}+3 f_{5}$ & $.5 u_{5}^{2}+u_{5}$ & 0.97 & 0.00 & 0.00 & - \\
\hline 6 & $f_{6}^{2}+2 f_{6}$ & $.5 u_{6}^{2}+u_{6}$ & 1.40 & 0.00 & 0.00 & - \\
\hline 7 & $.5 f_{7}^{2}+2 f_{7}$ & $.5 u_{7}^{2}+u_{7}$ & 3.11 & 0.00 & 0.00 & - \\
\hline 8 & $.5 f_{8}^{2}+2 f_{8}$ & $.5 u_{8}^{2}+u_{8}$ & 3.47 & 0.00 & 0.00 & - \\
\hline 9 & $f_{9}^{2}+5 f_{9}$ & $.5 u_{9}^{2}+u_{9}$ & 0.38 & 0.00 & 0.00 & - \\
\hline 10 & $.5 f_{10}^{2}+2 f_{10}$ & $.5 u_{10}^{2}+u_{10}$ & 5.75 & 0.00 & 0.00 & - \\
\hline 11 & $f_{11}^{2}+f_{11}$ & $.5 u_{11}^{2}+u_{11}$ & 4.46 & 0.00 & 0.00 & - \\
\hline 12 & $.5 f_{12}^{2}+2 f_{12}$ & $.5 u_{12}^{2}+u_{12}$ & 0.82 & 0.00 & 0.00 & - \\
\hline 13 & $.5 f_{13}^{2}+5 f_{13}$ & $.5 u_{13}^{2}+u_{13}$ & 0.52 & 0.00 & 0.00 & - \\
\hline 14 & $f_{14}^{2}$ & $.5 u_{14}^{2}+u_{14}$ & 4.41 & 0.00 & 0.00 & - \\
\hline 15 & $f_{15}^{2}+2 f_{15}$ & $.5 u_{15}^{2}+u_{15}$ & 0.00 & 0.00 & 0.00 & - \\
\hline 16 & $.5 f_{16}^{2}+3 f_{16}$ & $.5 u_{16}^{2}+u_{16}$ & 0.05 & 0.00 & 0.00 & - \\
\hline 17 & $.5 f_{17}^{2}+2 f_{17}$ & $.5 u_{17}^{2}+u_{17}$ & 4.41 & 0.00 & 0.00 & - \\
\hline 18 & $10 f_{18}$ & - & 5.00 & - & - & 10.89 \\
\hline 19 & $12 f_{19}$ & - & 10.00 & - & - & 11.59 \\
\hline 20 & $15 f_{20}$ & - & 5.00 & - & - & 11.96 \\
\hline
\end{tabular}

\section{Example 6}

Example 6 had the identical data to that in Example 5 except that we now assumed that the organization had capacities on its supply chain network activities where $\bar{u}_{a}=10$, for all $a \in L^{1}$. The complete data for Example 6 and the solution are given in Table 2. As can be seen from Table 2, links 13 and 15 now have positive associated flows although at very low levels.

\section{Example 7}

Example 7 had the same data as Example 6 except that we changed the probability distri- 
butions so that we now had:

$$
\begin{aligned}
P_{1}\left(\sum_{p \in P_{w_{1}}} x_{p}\right) & =\frac{\sum_{p \in P_{w_{1}}} x_{p}}{110}, \\
P_{2}\left(\sum_{p \in P_{w_{2}}} x_{p}\right) & =\frac{\sum_{p \in P_{w_{2}}} x_{p}}{120}, \\
P_{3}\left(\sum_{p \in P_{w_{3}}} x_{p}\right) & =\frac{\sum_{p \in P_{w_{3}}} x_{p}}{130} .
\end{aligned}
$$

The complete total cost data and solution for Example 7 are reported in Table 3 . The optimal supply chain network design for Example 7 has the topology given in Figure 3 since there are now positive flows on all the links. It is also interesting to note the increase in production volumes by the organization at its manufacturing plants; see $f_{1}^{*}, f_{2}^{*}$, and $f_{3}^{*}$ in Table 3, as compared to the analogous flows for Examples 5 and 6 in Tables 1 and 2, respectively. 
Table 3: Total Cost Functions and Solution for Example 7

\begin{tabular}{|c|c|c|c|c|c|c|}
\hline Link $a$ & $\hat{c}_{a}\left(f_{a}\right)$ & $\hat{\pi}_{a}\left(u_{a}\right)$ & $f_{a}^{*}$ & $u_{a}^{*}$ & $\omega_{a}^{*}$ & $\gamma_{a}^{*}$ \\
\hline 1 & $f_{1}^{2}+2 f_{1}$ & $.5 u_{1}^{2}+u_{1}$ & 4.23 & 0.00 & 0.00 & - \\
\hline 2 & $.5 f_{2}^{2}+f_{2}$ & $.5 u_{2}^{2}+u_{2}$ & 9.06 & 0.00 & 0.00 & - \\
\hline 3 & $.5 f_{3}^{2}+f_{3}$ & $.5 u_{3}^{2}+u_{3}$ & 8.61 & 0.00 & 0.00 & - \\
\hline 4 & $1.5 f_{4}^{2}+2 f_{4}$ & $.5 u_{4}^{2}+u_{4}$ & 2.05 & 0.00 & 0.00 & - \\
\hline 5 & $f_{5}^{2}+3 f_{5}$ & $.5 u_{5}^{2}+u_{5}$ & 2.18 & 0.00 & 0.00 & - \\
\hline 6 & $f_{6}^{2}+2 f_{6}$ & $.5 u_{6}^{2}+u_{6}$ & 3.28 & 0.00 & 0.00 & - \\
\hline 7 & $.5 f_{7}^{2}+2 f_{7}$ & $.5 u_{7}^{2}+u_{7}$ & 5.77 & 0.00 & 0.00 & - \\
\hline 8 & $.5 f_{8}^{2}+2 f_{8}$ & $.5 u_{8}^{2}+u_{8}$ & 7.01 & 0.00 & 0.00 & - \\
\hline 9 & $f_{9}^{2}+5 f_{9}$ & $.5 u_{9}^{2}+u_{9}$ & 1.61 & 0.00 & 0.00 & - \\
\hline 10 & $.5 f_{10}^{2}+2 f_{10}$ & $.5 u_{10}^{2}+u_{10}$ & 12.34 & 2.34 & 3.34 & - \\
\hline 11 & $f_{11}^{2}+f_{11}$ & $.5 u_{11}^{2}+u_{11}$ & 9.56 & 0.00 & 0.00 & - \\
\hline 12 & $.5 f_{12}^{2}+2 f_{12}$ & $.5 u_{12}^{2}+u_{12}$ & 5.82 & 0.00 & 0.00 & - \\
\hline 13 & $.5 f_{13}^{2}+5 f_{13}$ & $.5 u_{13}^{2}+u_{13}$ & 2.38 & 0.00 & 0.00 & - \\
\hline 14 & $f_{14}^{2}$ & $.5 u_{14}^{2}+u_{14}$ & 4.14 & 0.00 & 0.00 & - \\
\hline 15 & $f_{15}^{2}+2 f_{15}$ & $.5 u_{15}^{2}+u_{15}$ & 2.09 & 0.00 & 0.00 & - \\
\hline 16 & $.5 f_{16}^{2}+3 f_{16}$ & $.5 u_{16}^{2}+u_{16}$ & 2.75 & 0.00 & 0.00 & - \\
\hline 17 & $.5 f_{17}^{2}+2 f_{17}$ & $.5 u_{17}^{2}+u_{17}$ & 4.72 & 0.00 & 0.00 & - \\
\hline 18 & $10 f_{18}$ & - & 5.00 & - & - & 34.13 \\
\hline 19 & $12 f_{19}$ & - & 10.00 & - & - & 31.70 \\
\hline 20 & $15 f_{20}$ & - & 5.00 & - & - & 29.66 \\
\hline
\end{tabular}




\section{Remark}

In Tables 1, 2, and 3, there are no $\gamma_{a}^{*}$ values for links 1 through 17 since we assumed that the organization has no existing capacities. Furthermore, links 18, 19, and 20 are outsourcing links and they do not have any capacities. Hence, the associated capacity investment costs and the Lagrange multipliers are not needed in the formulation and solution.

\section{Summary and Conclusions}

In this paper, we developed an integrated framework for the design of supply chain networks for critical products such as vaccines, medicines, food, etc., which may be used in preparation (and response) to pandemics, disasters, attacks, etc. The model utilizes cost minimization within a system-optimization perspective as the primary objective and captures rigorously the uncertainty associated with the demand for critical products at the various demand points. In addition, the supply chain network design model allows for the investment of enhanced link capacities associated with such supply chain activities as manufacturing, storage, and distribution. Moreover, it allows for nonnegative initial capacities on the supply chain activities that the organization controls. The organization contracts the outsource product volumes at a fixed price. Finally, the model allows for the investigation of whether the product should be outsourced or produced in-house.

The methodology that we utilize for the formulation and solution of the supply chain network design model for critical needs is that of the theory of variational inequalities. The formulation allows for the implementation of an algorithm which yields very simple subproblems at each iteration, each of which can be solved explicitly and in closed form. Indeed, since the model makes use of continuous variables exclusively (as opposed to binary variables) and this feature enables effective and efficient solution of numerical problems based on this framework.

The framework developed here can be applied in numerous situations in which the goal is to produce and deliver a critical product at minimal cost so as to satisfy the demand at various demand points, as closely as possible, given associated penalties for under-supply (and, if also relevant, for over-supply, which we expect to be lower than the former). 
The model is discussed in the context of distinct applications, specifically, to vaccine production as well as to emergency preparedness and humanitarian logistics. The model is also illustrated with a spectrum of numerical examples for which the optimal product flows, investment capacities, as well as Lagrange multipliers associated with the constraints are computed.

This paper is a contribution to the literature of supply chain networks with a focus on design and humanitarian and healthcare applications.

\section{Acknowledgments}

The authors are indebted to the three anonymous reviewers and to the Associate Editor for helpful comments and suggestions on two earlier versions of this paper.

This research was supported by the John F. Smith Memorial Fund at the Isenberg School of Management. This support is gratefully acknowledged.

The first author also acknowledges support from the Rockefeller Foundation and its Bellagio Center Program, which made possible the conference "Humanitarian Logistics: Networks for Africa," which took place at the Bellagio Center on Lake Como, Italy in May 2008. Finally, the first author acknowledges Professor Manfred Fischer, who hosted her seminar on supply chain vulnerabilities and synergies in March 2009 at the Vienna University of Business and Economics. Some of the ideas in this paper were inspired by subsequent discussions.

\section{References}

Altay N (2008) Issues in disaster relief logistics. In: Gad-el-Hak M (ed) Large-scale disasters: Prediction, control, and mitigation. Cambridge University Press. Cambridge, United Kingdom

Balcik B, Beamon B (2008) Facility location in humanitarian relief. International Journal of Logistics: Research and Applications 11: 101-121

Barbara M, Gillis J (2005) Wal-Mart at forefront of hurricane relief. The Washington Post September 6 . 
Beamon BM, Kotleba SA (2006) Inventory modeling for complex emergencies in humanitarian relief operations. International Journal of Logistics: Research and Applications 9: 1-18

Beckmann MJ, McGuire CB, Winsten CB (1956) Studies in the economics of transportation. Yale University Press, New Haven, Connecticut

Bertsekas DP, Tsitsiklis JN (1989) Parallel and distributed computation - Numerical methods. Prentice Hall, Englewood Cliffs, New Jersey

Boyce DE, Mahmassani HS, Nagurney A (2005) A retrospective on Beckmann, McGuire, and Winsten's Studies in the economics of transportation. Papers in Regional Science 84: 85-103

Cowell A, Otterman S (2010) Relief groups seek alternative routes to get aid moving. The New York Times. January 15.

CNN.com (2009) Global swine flu deaths top 1,100. August 5. Available online at: http://edition.cnn.com/2009/HEALTH/08/05/swine.flu.deaths/

Coleman MS, Sangrujee N, Zhou F, Chu S (2005) Factors affecting U.S. manufacturers' decisions to produce vaccines. Health Affairs 24: 635-642

Dafermos SC, Sparrow FT (1969) The traffic assignment problem for a general network. Journal of Research of the National Bureau of Standards 73B: 91-118

Development Initiatives (2006) Global humanitarian assistance 2006 report. Available online at: http://www.globalhumanitarianassistance.org/pdfdownloads/GHA\%202006.pdf

Dong J, Zhang D, Nagurney A (2004) A supply chain network equilibrium model with random demands. European Journal of Operational Research 156: 194-212.

Dupuis P, Nagurney A (1993) Dynamical systems and variational inequalities. Annals of Operations Research 44: 9-42

Friesz TL, Editor (2007) Network science, nonlinear science and infrastructure systems. 
Springer, Berlin, Germany

Guidotti TL (2006) Hurricane Katrina: An American tragedy. Occupational Medicine 56: 222-224

International Strategy for Disaster Reduction (2006) Press Release. January 30, 2006. Available online at: http://www.unisdr.org/eng/media-room/press-release/2006/ PR-200602-Disasters-increase-18-per-cent-2005-but-death-rates-drop.pdf

Klein JO, Myers MG (2006) Strengthening the supply of routinely administered vaccines in the United States: Problems and proposed solutions. Clinical Infectious Diseases 42: S97-S103

Miller T, Friesz TL, Tobin RE (1996) Equilibrium facility location on networks. Springer, Berlin, Germany.

Mowery DC, Mitchell V (1995) Improving the reliability of the U. S. vaccine supply: An evaluation of alternatives. Journal of Health Politics, Policy and Law 20: 973-1000

Nagurney A (1993) Network economics: A variational inequality approach. Kluwer Academic Publishers, Dordrecht, The Netherlands

Nagurney A (2006) Supply chain network economics: Dynamics of prices, flows and profits. Edward Elgar Publishing, Cheltenham, England

Nagurney A (2008) Final report to the Rockefeller Foundation: Humanitarian logistics: Networks for Africa Bellagio Center Conference. May 2008, Bellagio, Italy

Nagurney A (2009a) A system-optimization perspective for supply chain network integration: The horizontal merger case. Transportation Research E 45: 1-15

Nagurney A (2009b) Formulation and analysis of horizontal mergers among oligopolistic firms with insights into the merger paradox: A supply chain network perspective. Computational Management Science in press

Nagurney A (2010) Supply chain network design under profit maximization and oligopolistic 
competition. Transportation Research E 46: 281-294

Nagurney A, Dong J, Zhang D (2002) A supply chain network equilibrium model. Transportation Research E 38: 281-303

Nagurney A, Dupuis P, Zhang D (1994) A dynamical systems approach for network oligopolies and variational inequalities. Annals of Regional Science 28: 263-283

Nagurney A, Matsypura D (2005) Global supply chain network dynamics with multicriteria decision-making under risk and uncertainty. Transportation Research E 41: 585-612

Nagurney A, Qiang, Q (2009) Fragile networks: Identifying vulnerabilities and synergies in an uncertain world. John Wiley \& Sons, Hoboken, New Jersey

Nagurney A, Takayama T, Zhang D (1995) Projected dynamical systems modeling and computation of spatial network equilibria. Networks 26: 69-85

Nagurney A, Woolley T (2010) Environmental and cost synergy in supply chain network integration in mergers and acquisitions. In: Ehrgott M, Naujoks B, Stewart T, Wallenius J (eds) Sustainable energy and transportation systems, Proceedings of the 19th International Conference on Multiple Criteria Decision Making, Lecture notes in economics and mathematical systems. Springer, Berlin, Germany, 51-78

Nagurney A, Woolley T, Qiang, Q (2009) Multiproduct supply chain horizontal network integration: Models, theory, and computational results. International Transactions in Operational Research, in press

Nagurney A, Zhang D (1996) Projected dynamical systems and variational inequalities with applications. Kluwer Academic Publishers, Norwell, Massachusetts

Qiang Q, Nagurney A, Dong J (2009) Modeling of supply chain risk under disruptions with performance measurement and robustness analysis. In: Wu T, Blackhurst J (eds) Managing supply chain risk and vulnerability: Tools and methods for supply chain decision makers. Springer, London, England, 91-111 
Reuters.com (2009) Large-scale H1N1 vaccine production begins - Sanofi, June 25. Available online at: http://www.alertnet.org/thenews/newsdesk/N25297316.htm

Treanor J (2004) Weathering the influenza vaccine crisis. New England Journal of Medicine 351: $2037-2040$

World Health Organization (2009) Rethinking the vaccine supply chains. Available online at: http://www.who.int/immunization_delivery/systems_policy/optimize/en/index.html

Zhang D (2006) A network economic model for supply chain vs. supply chain competition. Omega 34: 283-295

Zhang D, Dong J, Nagurney A (2003) A supply chain network economy: modeling and qualitative analysis. In: Nagurney A (ed) Innovations in financial and economic networks. Edward Elgar Publishing, Cheltenham, England, 197-213 